\title{
Combining new data collection tools and improved beam delivery on the macromolecular crystallography beamline I04 at Diamond Light Source
}

Ralf Flaig, Pierpaolo Romano, Jonathan Blakes Chris Bloomer, Graham Duller, Sandira Gayadeen, Michael Hillman, James O’Hea, Geoff Preece, Graeme Winter, David R. Hall

Diamond Light Source, Harwell Science and Innovation Campus, Chilton, Didcot, OX11 ODE, UK.

\author{
Author Email: ralf.flaig@diamond.ac.uk
}

Diamond Light Source [1] currently operates five beamlines for macromolecular crystallography (MX) and soon seven beamlines will serve the MX user community [2]. I04, a widely tuneable (5-25 $\mathrm{keV}$, core range 6-17 keV) SAD/MAD station [3] is constantly evolving with the aim to provide the user with state of the art tools for data collection, especially to enable structure solution from increasingly difficult and challenging samples.

Since early 2015 the beamline is using an array of compound refractive lenses in a transfocator like device allowing the user to quickly change the beam size from 10 (h) $\times 5$ (v) up to 110 (h) x 100 (v) microns over a wide energy range and thus be better able to match the beam size to the crystal size or be able to select more precisely the best part of the crystal. Using these small beam sizes, especially with small sample sizes, requires much more stringent schemes in terms of reliable beam delivery with respect to beam intensity and position than were envisaged for the original beamline design, including the monochromator and beam diagnostics. We therefore have started a programme to improve beam delivery and have recently installed a new double crystal monochromator (DCM) which was designed inhouse based on existing proven technology and experience gained from other designs. The aim is to provide a system that is more stable, also taking into account possible future changes e.g. an increase in ring current, a lower emittance storage ring lattice and a new insertion device with higher power output. Alongside this, we have installed new beam position and intensity monitors that we are currently commissioning to be used in conjunction with a beam delivery feedback system.

We are also looking into an improved goniometry and sample test system. As part of this we are planning the installation of the SmarGon multi-axis goniometer, which will enable better and faster sample centring, improved helical scan performance and provide users with new scientific capabilities for multi-sweep data collection strategies using multiple crystal orientations. Alongside these upgrades in beamline hardware we are also working on the development of the user software interface in order to streamline the user experiments.

\section{References}

[1] http://www.diamond.ac.uk

[2] http://www.diamond.ac.uk/Mx.html

[3] http://www.diamond.ac.uk/Beamlines/Mx/I04.html

Keywords: Diamond Light Source, macromolecular crystallography, beamlines, experimental phasing, data collection, multi-axis goniometry, monochromator, beam diagnostics, feedback 